Relations industrielles

Industrial Relations

\title{
L'autorité dans l'entreprise
}

\section{Victor Morency}

Volume 5, numéro 6, mars 1950

URI : https://id.erudit.org/iderudit/1023349ar

DOI : https://doi.org/10.7202/1023349ar

Aller au sommaire du numéro

Éditeur(s)

Département des relations industrielles de l’Université Laval

ISSN

0034-379X (imprimé)

1703-8138 (numérique)

Découvrir la revue

Citer cet article

Morency, V. (1950). L'autorité dans l'entreprise. Relations industrielles /

Industrial Relations, 5(6), 56-57. https://doi.org/10.7202/1023349ar

Tous droits réservés (C Département des relations industrielles de l’Université Laval, 1950
Ce document est protégé par la loi sur le droit d'auteur. L'utilisation des services d'Érudit (y compris la reproduction) est assujettie à sa politique d'utilisation que vous pouvez consulter en ligne.

https://apropos.erudit.org/fr/usagers/politique-dutilisation/ 


\section{L'AUTORITÉ DANS L'ENTREPRISE}

\section{Victor MORENCY}

La légitimité des titres à l'autorité dans l'entreprise à propriétaire unique ou à caractère familial est basée sur le droit de chacun à gérer ses biens et sur un argument psychologique. Sauf de rares exceptions, l'investissement des capitaux dans de telles entreprises constitue un risque; or, il est dans l'ordre des exigences psychologiques des apporteurs de capitaux d'avoir cette garantie, qu'ils jugent nécessaire, de désigner eux-mêmes l'autorité dans l'entreprise, sans quoi ils renonceraient à risquer leurs argents.

Cet argument a beaucoup moins de force en a-t-il vraiment ? - dans le cas des sociétés anonymes. Les actionnaires, qui sont les détenteurs des capitaux dans ces sociétés ont, en théorie, le pouvoir de nommer l'autorité dans l'entreprise; mais il suffit de comparer le nombre des actionnaires qui prennent part aux assemblées générales à celui de l'ensemble des actionnaires pour estimer à de justes proportions le rôle qu'ils jouent effectivement.

C'est pourquoi, dans ces sociétés, le choix de l'autorité étant laissé à un petit nombre, l'argument psychologique suivant lequel les détenteurs de capitaux n'investissent qu'à la condition de nommer l'autorité est faussé. Car c'est ce petit nombre, qui, par défaut, arrive à détenir une autorité perpétuelle.

On peut se demander si ce n'est pas le sens de l'affirmation de Pie XII, le 7 mai 1949, devant l'UNIAPAC: «Le propriétaire des moyens de production, quel qu'il soit - propriétaire particulier, association d'ouvriers ou fondation - doit, toujours dans les limites du droit public de l'économie, rester maître de ses décisions économiques », puisqu'il prenait la peine d'indiquer ce qu'il entendait par «propriétaire des moyens de production».

Il est important de distinguer, dans l'autorité, sa raison d'être même et le pouvoir qu'elle comporte. Celui qui, par voie d'hérédité ou de nomination, dirige une entreprise, exerce un pouvoir qui «n'est souvent, comme l'a écrit Jean Predseil, qu'un fait matériel plus ou moins accidentel $\gg .{ }^{1}$ Mais ce pouvoir, s'il est mal fondé et mal exercé, empêchera l'entreprise d'atteindre à sa fin propre qui est son bien commun. C'est donc par une grande valeur morale, un souci éprouvé de justice et des connaissances techniques certaines que le

(1) Predseil, Jean «Participation ouvrière et autorité patronale », Industrie, oct. 1948, p. 627. chef d'entreprise pourra s'assurer la confiance et l'acceptation de ses collaborateurs sans avoir recours à la coercition. Il ne faut pas se faire d'illusions. A la minute où l'ouvrier met en doute la moralité, l'esprit de justice et les connaissances du patron, son obéissance cesse d'être libre et consentie, donc d'être efficace, pour devenir un acte de soumission forcée qu'à la première occasion il transformera en un geste de ressentiment ou de rébellion.

C'est ce que soulignait, en 1945, l'assemblée des cardinaux et archevèques de France: «Pour réaliser ces progrès sociaux (la promotion ouvrière), des réformes de structures sont nécessaires. Nous sommes prêts à les accueillir. Mais pour quelles soient plus efficaces, il importe que l'unité de commandement et que l'autorité du chef d'entreprise, fondés sur la compétence et la moralité, soient pleinement reconnus, qu'il ne soit pas porté atteinte aux droits légitimement acquis, qu'il soit tenu compte avec prudence des capacités de l'économie nationale et que tout esprit de violence soit écarté ».

Puisque la Providence a donné à la plupart des chefs d'entreprises le pouvoir qui découle de l'autorité, sans qu'ils y soient parfois pour grandchose, il importe qu'ils sachent l'exercer d'une façon qui transforme l'obéissance de leurs employés en un geste de collaboration spontané.

Lorsque l'on parle de participation à l'autorité du chef d'entreprise et que l'on conteste au patron l'exclusivité de ses titres à la direction, on n'exprime pas nécessairement des idées marxistes. L'entreprise est une communauté humaine où l'autorité n'est justifiée que par le service qu'elle rend en assurant le bien commun. Trop de patrons n'ont cependant exercé leur autorité qu'en vue de leurs intérêts propres au détriment des travailleurs et, par conséquent, du bien de l'entreprise; de ce chef, ils ont cessé de remplir légitimement leur fonction.

Que faire, dans ce cas, en face de revendications ouvrières très justes ? Amener le patronat à maintenir ses positions acquises au cours du siècle dernier, ce qui équivaut, par contre-coup, à s'acheminer vers l'entreprise nationalisée de l'Etat socialiste ? Ou encore, ce qui est beaucoup plus sage, entreprendre ensemble, patrons et ouvriers, l'instauration de la démocratie économique chrétienne demandée dans les enseignements pontificaux? 
«Aujourd'hui, a écrit Thomas Lhoest, il n'y a que deux solutions possibles: celle d'accepter les transferts progressifs des responsabilités aux masses syndicales révolutionnaires, qui devront en faire ensuite un groupe de dirigeants durs et implacabes; ou bien celle de développer des transferts progressifs et ordonnés de la responsabilité personnelle à tous ceux qui participent à la production, courant ainsi leur part de risques. »

«Cette seconde solution est sans doute, déclarait le docteur Vittorio Vaccari au dernier Congrès de l'A.P.I., dans l'esprit des principes de la doctrine sociale chrétienne et correspond aux directives données par le Saint-Père aux chefs d'entreprises. »

Un article publié récemment dans la revue américaine Fortune indique bien comment on sait, dans un pays matérialiste comme les Etats-Unis, envisager bien en face le problème de l'autorité dans lentreprise et en arriver à une solution également réaliste. L'auteur de cet article, Neil Chamberlain, du Labor and Management Center de l'université Yale, répond à la question «What is Management's Right to Manage ?»

«En 1851, dit-il, le Journal of Commerce de New York écrivait: 'Nous n'abandonnerons pas le contrôle de nos affaires aux dictées d'un pouvoir constitué de lui-même hors de nos bureaux' en matière d'heures de travail et d'apprentissage. Aujourd'hui ces questions sont chose commune dans les conventions collectives. En 1945, continue-t-il, les aciéries américaines refusèrent aux syndicats ouvriers leur mot à dire dans les plans de pension des compagnies. Aujourd'hui, ces plans sont couramment inclus dans les contrats collectifs...

«La difficulté dans le cas de la propriété, en temps qu'elle concerne l'autorité, c'est qu'elle ne donne de pouvoir que sur les choses... Mais dans l'entreprise, poursuit-il, les gens ne peuvent être commandés et dirigés que de leur libre consentement... Il n'y a aucune force légale pour obliger les travailleurs à coopérer... La définition des termes de la coopération est laissée directement aux intéressés. Et il n'y a rien dans la loi qui puisse empêcher le syndicat de demander, comme prix de la coopération de ses membres, une voix en des matières qui étaient auparavant déterminées par la direction seule. Comme le droit de propriété ne donne pas un pouvoir sur les autres, l'employeur pourra alors trouver essentiel de partager son autorité comme moyen de maintenir la valeur de la production...

«Dans ce cas, conclut-il, les syndicats ne seraient pas considérés comme quelque chose hors de la structure et du processus de la direction, mais, en fait, une partie intégrante ». ${ }^{3}$

Reconnaissons que l'ouvrier, dans la sphère propre de son travail, est habituellement beaucoup plus compétent que le patron. Le temps et la pratique lui ont donné cette supériorité. Cette compétence ne s'épanouira pas cependant si le chef d'entreprise se borne à l'utiliser par le commandement. Mais qu'il fassc appel à ses avis, à ses suggestions au plan de son travail, et la métamorphose s'opère. D'un mécanisme qu'il était dans l'ensemble de l'organisation productive, l'ouvrier, qu'il soit travailleur manuel ou technicien professionnel, devient alors une unité pensante qui contribue, comme seul l'homme peut le faire, à faire vivre et grandir l'entreprise. Le chef d'entreprise affaiblit son autorité quand, voulant entrer dans les détails de son commandement, il découvre à l'ouvrier, dans la sphère propre du travail de celui-ci, des connaissances techniques insuffisantes. Se sentant supérieur dans ces occasions, l'ouvrier perd confiance et développe un esprit de critique.

Aussi, après avoir écouté ses avis, recueilli ses suggestions, il faudra faire un deuxième pas: celui de renseigner l'ouvrier sur son rôle dans la marche de l'entreprise. «Les hommes, a écrit Jean Lannoye, doivent le moins possible subir l'autorité, mais bien l'accepter par un acte libre, parce qu'ils en découvrent concrètement la nécessité, les fondements, les limites et les préoccupations désintéressés. » ${ }^{*}$ C'est ainsi que le chef d'entreprise pourra faire preuve d'intelligence et d'humanité.

Enfin - et c'est là une suggestion audacieuse pour réaliser la démocratie économique dans l'entreprise - il serait bon, en troisième lieu, de demander un avis à un comité formé des représentants de chaque échelon dans la hiérarchie de l'entreprise sur la nomination du chef direct de cet échelon. Cet avis cependant ne lierait pas la direction.

Ces réformes exigent certes une évolution sensible dans les esprits et dans les consciences, elles comportent aussi un risque, mais nous croyons que ce risque est moins grand que celui de ne pas le tenter. Pour ce faire, patrons comme ouvriers devraient renoncer à leur esprit de lutte et reconnaître la véritable fonction de l'entreprise qui est "d'intégrer chaque homme dans une communauté vivante où il occupera la place que ses mérites et ses dispositions lui confèrent naturellement ».

(3) Chamberlain, Neil, «What is Management's Right to Manage? 》, Fortune, 1939, pp. 68-69-70.

(4) LANNoye, Jean, «L'autorité dans l'entreprise », Bulletin social des industriels, janv. 1949. 